(C) 1983 IEEE. Personal use of this material is permitted. However, permission to reprint/republish this material for advertising or promotional purposes or for creating new collective works for resale or redistribution to servers or lists, or to reuse any copyrighted component of this work in other works must be obtained from the IEEE.

IEEE Transactions on Nuclear Science, Vol. NS-30, No. 4, August 1983

A SECOND HARMONIC (6-16 MHZ) RF SYSTEM WITH FEEDBACK-REDUCED GAP IMPEDANCE FOR ACCELERATING FLAT-TOPPED BUNCHES IN THE CERN PS BOOSTER

J.M. Baillod, L. Magnani, G. Nassibian, F. Pedersen and W. Weissflog CERN, 1211 Geneva 23, Switzerland

Introduction and Summary

Since all experimental and heoretical evidence indicates that the beam intensity in the PSB is at present 1 imited by the Laslett $Q$ shift after trapping, a second harmonic accelerating system is being added with the aim of producing flat-topped bunches. Calculations indicate that the resulting improvement in bunching factor should permit an intensity increase of 25 to $40 \%$. Additionally, the bucket area is increased by 25 to $45 \%$ depending on intcnsity.

A general description of the new cavities, which operate over a frequency range of $6-16 \mathrm{MHz}$ with a maximum gap voltage of $8 \mathrm{kV}$, is given together with a description of associated power amplifiers and low level electronics. A notable feature is the use of a fast feedback system to reduce the gap impedance seen by the beam by an order of magnitude, incorporating a $130 \mathrm{~W}$ wide-band transistor amplifier with a total delay of about 5 ns.

Initial performance with beam of the first system installed confirms theoretical expectations in terms of bunching factor and increased intensity 1 imit. An expected improvement in beam stabilization by Landau damping from the strongly non-linear accelerating wave shape did not occur; on the contrary, Landau damping is lost even at low intensities, and harmful longitudinal bunched-beam modes of orders as high as sextupole $(m=3)$ and decapole $(m=5)$ have been observed and partly cured.

Bunching Factor, Bucket Area and Landau Damping

The applied focusing wave shape is

$$
\mathrm{V}(\phi)-\mathrm{v}_{0}=\mathrm{v}_{1}\left(\sin \phi-\mathrm{a}_{2} \sin 2\left(\phi-\phi_{\mathrm{S}}\right)-\sin \phi_{\mathrm{S}}\right)
$$

where $e v_{0}=e V_{1} \sin \phi_{S}$ is the energy gain per turn, $v_{1}$ the amplitude of the fundamental voltage, $\phi_{S}$ the phase of the synchronous particle, and $a_{2}=v_{2} / v_{1}$ the relative amplitude of the second harmonic voltage. The second harmonic has been phased so as to provide zero voltage for $\phi=\phi_{s}$.

If we assume an elliptic energy distribution, the stationary line density $\lambda(\phi)$ is proportional to the potential which is the integral of the focusins wave shape above, and can thus be found analytically.

For a $<0.5 \cos \phi_{\mathrm{S}}$ the 1 ine density has one peak for $\phi=\phi_{s}$, while for $a_{2}>0.5 \cos \phi_{s}$ there will be two peaks at $\phi=\phi_{\alpha}$ and $\phi=\phi_{\beta}$, both values are roots of $V(\phi)-V_{0}=0$. For $\phi_{S}=0$ the bunching factor for full buckets can be expressed analytically as a function of $a_{2}$, while for $\phi_{s} \frac{1}{\neq} 0$ and $a_{2}>0.5 \cos \phi_{s}$ the bunching factor must be determined numerically, as the roots $\phi_{\alpha}$ and $\phi_{B}$ must be found by iteration.

$B_{F}\left(a_{2}\right)=\left\{\begin{array}{l}\frac{1}{2}+\frac{a_{2}}{4} \text { for } 0 \leq a_{2} \leq 0.5 \\ \left(1+\frac{a_{2}}{2}\right) /\left(1+\frac{1}{4 a_{2}}+a_{2}\right) \text { for } a_{2}>0.5 .\end{array}\right.$

Surprisingly, the optimum voltage ratio is $a_{2}=1$, or twice the value which produces maximally flat-topped bunches: $a_{2}=0.5$. The optimum is, however, very flat, and by choosing $a_{2}=0.67$ on $1 \mathrm{y}$ about $3 \%$ lower $\mathrm{B}_{\mathrm{F}}$ 's result.

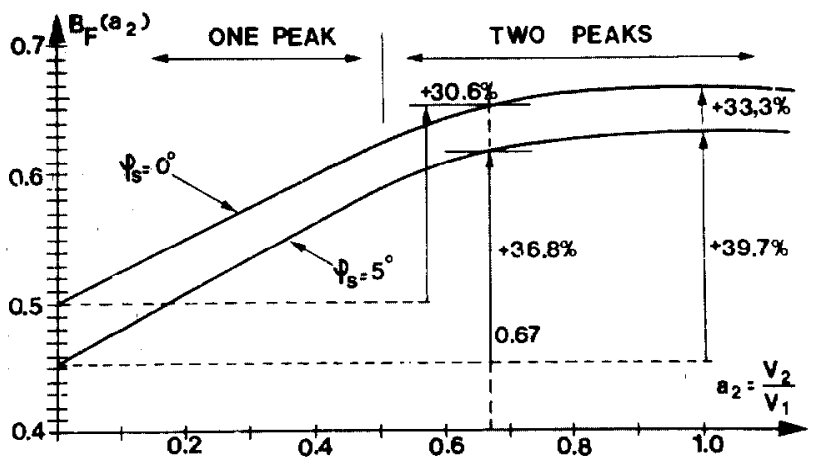

Fig. 1: Bunching factor versus $a_{2}$

As $\mathrm{V}_{1}=12 \mathrm{kV}$ and $\mathrm{f}_{1}=3$ to $8 \mathrm{MHz}$, we need $\mathrm{V}_{2}=8 \mathrm{kV}$ and $\mathrm{f}_{2}=6$ to $16 \mathrm{MHz}$ to obtain an almost optimal in crease in bunching factor (Figs. 1 and 2).

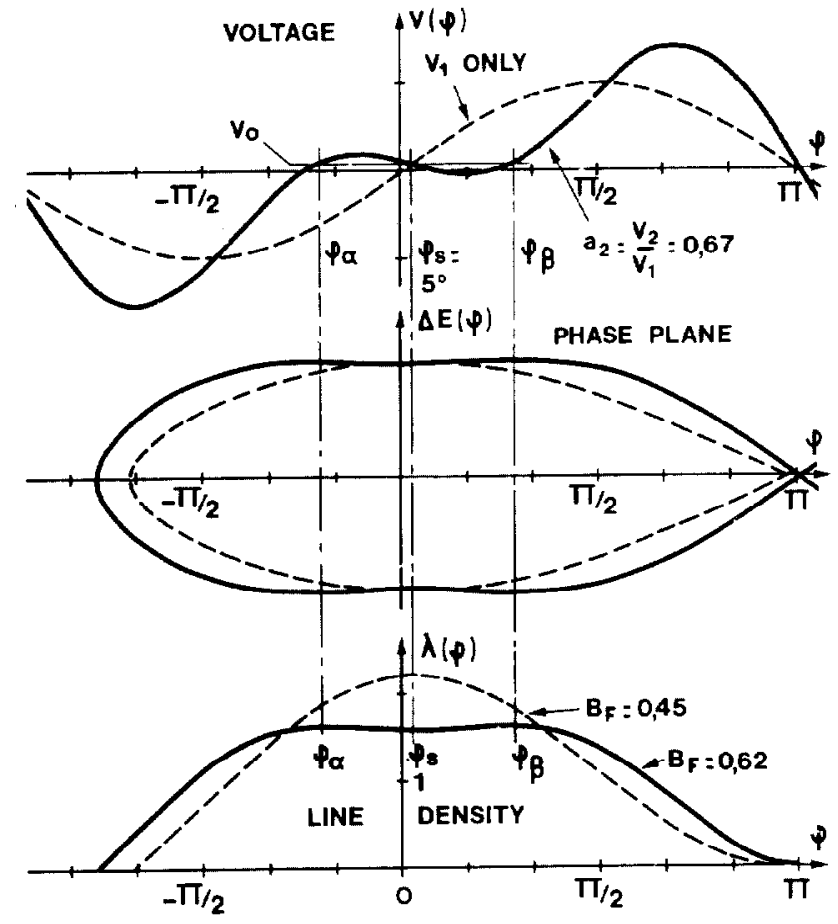

Fig. 2: Accelerating wave shape, bucket shape, and line density

For the elliptic energy distribution the bucket area (Fig. 3) including space charge effects is easy to calculate as space charge does not change the shape of the bucket, only its height ${ }^{1}$.

From the synchrotron frequency distribution (Fig. 4) with the second harmonic voltage added it is seen that the highly non-linear bucket provides plenty of frequency spread and thus Landau damping for amplitudes up to about 1.5 radians. For a full bucket, however, the flat maximum near 2 radians turns out to be particularly harmful as a strong density gradient is normally found in this region of the phase plane, resulting in substantially lower thresholds for longitudinal instabilities. 


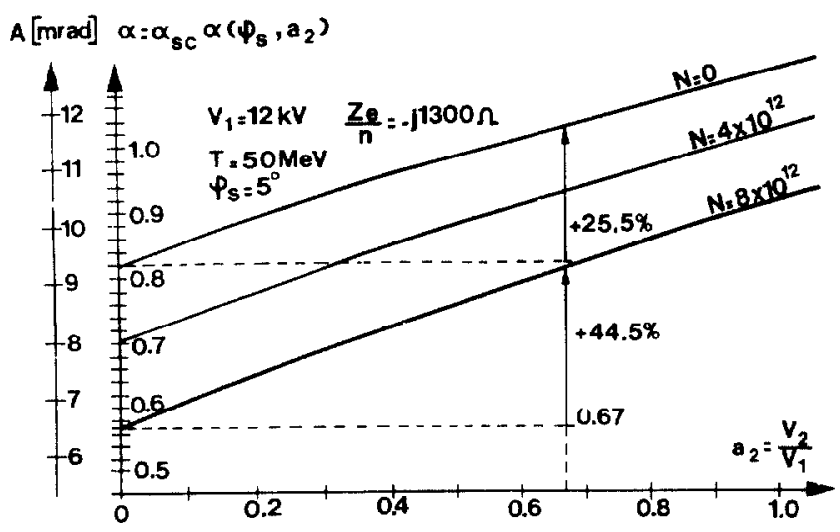

Fig. 3: Bucket area versus second harmonic voltage

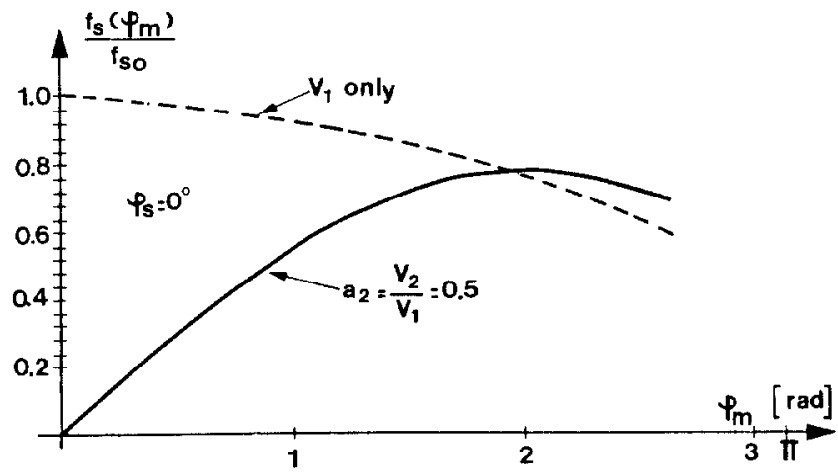

Fig. 4: Synchrotron frequency versus amplitude ${ }^{2}$

\section{Cavity and Amplifier Design}

The relatively small distance $(360 \mathrm{~mm}$ ) between the four superposed rings of the PSB imposes rather severe constraints on the design of the accelerating cavities and their associated final RF power stages, Figs. 5 and 6. Closed circuit air cooling is used for both, and their design generally follows that of the fundamental system ${ }^{3}$. For the 6-16 $\mathrm{MH}^{2}$ frequency range a different type of ferrite was, however, necessary, and Philips $4 \mathrm{M}$ was chosen.

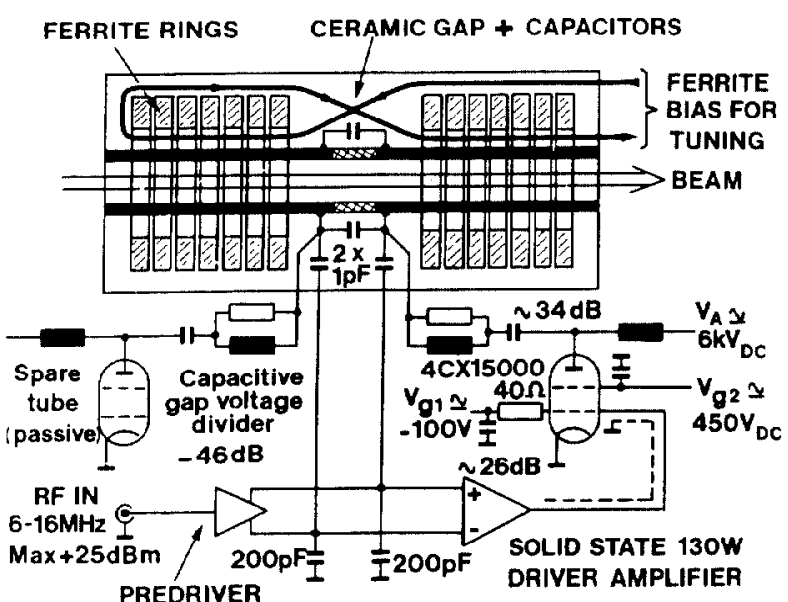

Eig. 5: Simplified schematic of cavity and amplifier

In order to ubtain the somewhat higher bias field required by this material, each cavity has four figureof-eight loops which carry the bias current and also provide RF coupling between the two ferrite cores, Fig. 6 .
The anode of a 4CX15000 tetrode is connected to each side of the gap vis a DC blocking condenser and a parallel RL combination which helps to damp some secondary resonances of the cavity and amplifier. A further damping of these resonances was obtained by connecting series $\mathrm{KC}$ circuits between nominally 'cold' points of the bias loops and the cavity walls.

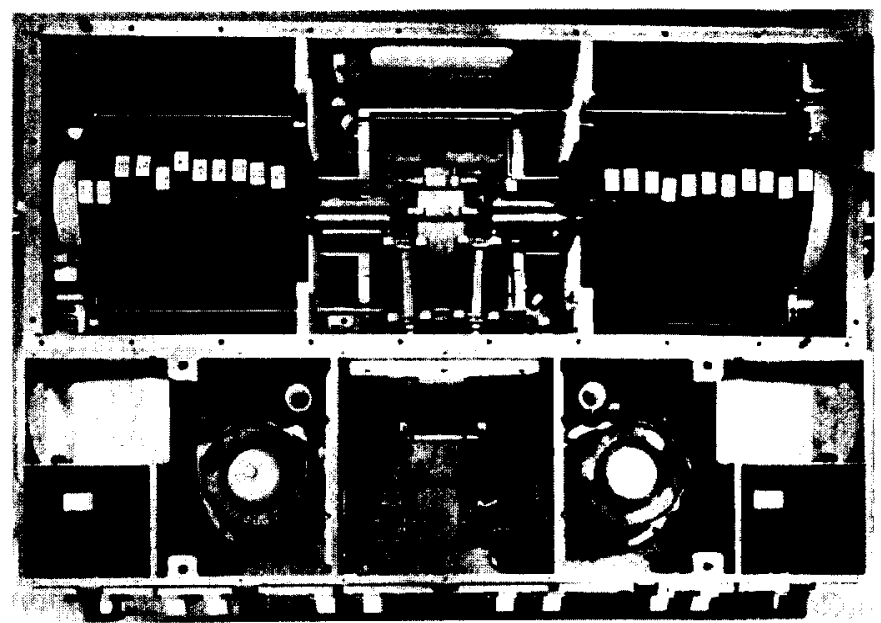

Fig. 6: Cavity and amplifier assembly

In normal operation only one tetrode is active, the other serving as a spare while maintaining capacitive halance.

In order to eliminate instabilities of the beam, cavity, and its associated loops ${ }^{4}$ under conditions with high relative beam loading, the final tube is driven by a feedback amplifier, whose inputs are connected to the gap via capacitive voltage dividers $(1: 200)$, thus forming a feedback loop with an open loop gain of 14-22 dB $(5-12 x)$, which reduces the effective impedarice by the same factor. This technique has previously been successfully used in the $\mathrm{ISR}^{5}$, and in the AA.

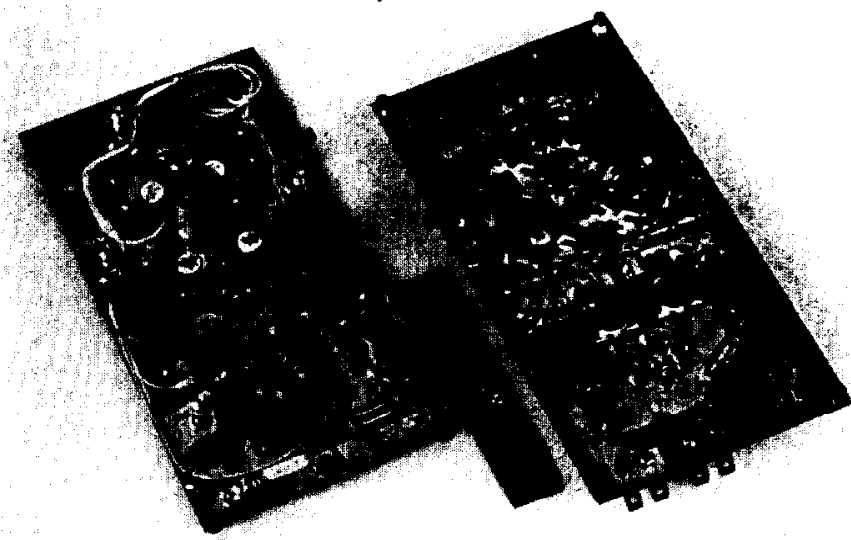

Fig. 7: Solid state driver amplifier

A linear, broadband solid state amplifier ( $F$ 'ig. 7) utilizing VMOS RF power transistors was designed specifically for this purpose with special attention to the very short propagation delay $(5 \mathrm{~ns})$ required to ensure loop stability over the 5-17 $\mathrm{MHz}$ frequency range. The input stage gate capacitance and predriver output capacitance form the $200 \mathrm{pF}$ bottom capacitor in the gap voltage divider. Power is $130 \mathrm{~W}$ and bandwidth $150 \mathrm{MHz}$.

\section{Low Leve1 Electronics}

Three control loops control relative phase, amplitude, and tune of the second harmonic cavity, Fig. 8 . 
The tuning loop acquires the tuning phase from inside the fast feedback loop which ensures automatic compensation of the reactive part of the beam loading and thus minimum tube power. The transistorized tuning power amplifier is controlled by the tuning loop amplifier which contains a compensation of the rather non-linear current-frequency relationship of the cavity to ensure reasonably constant loop gain, and an integrator which eliminates the need for feedback-forward compensation.

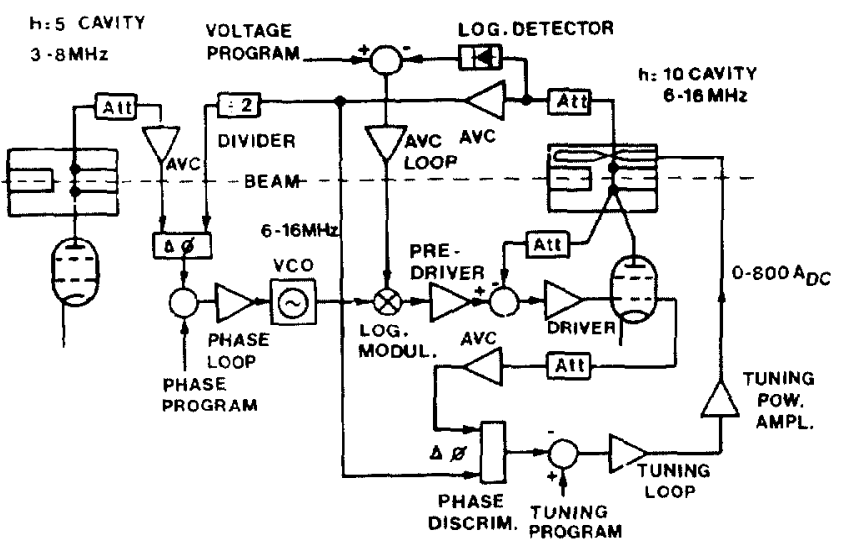

Fig. 8: Block diagram, low-level electronics

The voltage loop (AVC loop) acquires the gap level through a logarithmic detector to ensure a large dynamic range, and controls the level applied to the predriver through a logarithmic modulator capable of an equally large range of modulation. The voltage program is derived from a computer-controlled function generator.

The modulator is driven by a 6 to $16 \mathrm{MHz} \mathrm{VCO}$, which is controlled by a Phase-Locked Loop (PLL), which gets its phase references from the gaps of the two cavities through paths of equal propagation delays. The phase program is generated in an analog unit from signals representing $\mathrm{dB} / \mathrm{dt}$ and $\mathrm{V}_{1}$.

\section{Initial Experimental Results}

With $\mathrm{V}_{2}=6-8 \mathrm{kV}$ applied, a fast gruwing longitudinal instability developed immediately after trapping and resulted in a $50 \%$ beam loss. From its frequency, number of nodes, and bunch-to-bunch phase shift, the mode could be identified as an in-phase, sextupole mode $(n=0, m=3)$, Fig. 9 left.

The fact that it is an $n=0$ mode indicates that it is a generalized Kobinson type interaction; generalized so as to include feedback loops ${ }^{4}$ and higher order modes $^{7}(\mathrm{~m}>1)$. As the e-folding time $(\tau \approx 1 \mathrm{~ms})$ is independent of intensity, it became clear that the feedback path consists of the phase $100 p^{8}$ for the fundamental cavity, which compares the phase of that cavity with the fundamental of the beam signal, followed by the phase loop described above for the second harmonic cavity, which has a strong effect on the sextupole mode due to the higher form factor ${ }^{7}$ at this frequency.

An active damping of this mode was tried by detecting the phase modulation of the loth harmonic of the beam signal relative to the $h=10$ cavity with a mixer, and feeding this signal through an appropriate filter into the phase loop of the second harmonic cavity.

This was partly successful, the sextupole mode was damped, but a decapole mode $(\mathrm{n}=0, \mathrm{~m}=5, \mathrm{Fig}, 9 \mathrm{right})$ developed, except for the highest gain settings where stable bunches were obtained, Fig. 10, and a record intensity of $1.08 \times 10^{13}$ was captured in one ring. The
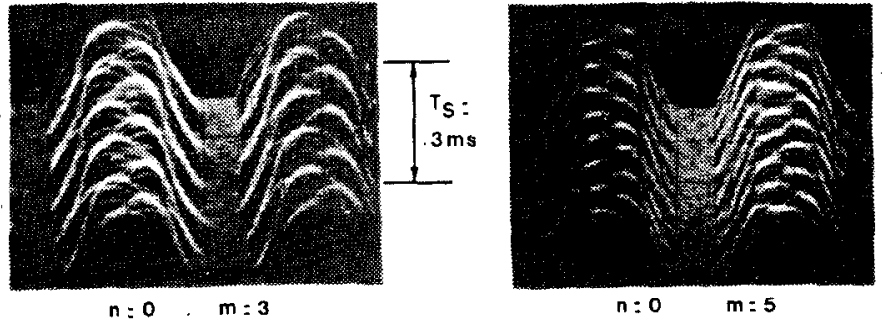

Fig. 9: Sextupole and decapole modes

sextupole/decapole damping system did not work efficiently to the end of the cycle, but nevertheless $8.5 \times$ $10^{12}$ could be accelerated to $800 \mathrm{MeV}$ by programming the second harmonic voltage.

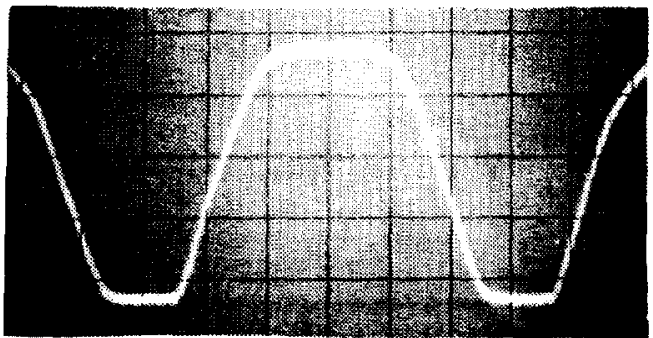

Fig. 10: Bunch shape, $N=8.5 \times 10^{12}, v_{1}=12 \mathrm{kV}, V_{2}=3 \mathrm{kV}$

Even at low intensities, say $10^{12}$, it turned out to be necessary to use all existing longitudinal damping systems, namely coupled mode active damping ${ }^{7}(n=1$ to $4, m=1,2$ and 3$)$, and Hereward damping $(n=0, m=2$ and 4). The in-phase quadrupole mode $(n=0, m=2)$ interacts very weakly at low intensity, so we studied this mode with ils active damping off, and even at low intensity it hardly showed any sign of Landau damping. The observed frequency corresponds well to the peak in Fig. 4, about $80 \%$ of the small amplitude synchrotron frequency with no second harmonic applied.

To conclude, adding the second harmonic cavities to the Booster rings increases the bunching factor and thus the intensity limit as expected, so that $10^{13}$ protons per ring does not seem out of reach. There is, however, a region in the phase plane with little or no Landau damping which makes the continued use of active feedback necessary, and results in a high complexity as we now have 11 feedback loops per ring associated with $\mathrm{RF}$ control and longitudinal mode damping.

\section{Acknowledgements}

The authors wish to thank R. Cornillon, J. Fopma, N. Pearce and C. Menot for their contributions, K.H. Reich for his support and encouragement and G. Gelato for measurements of bunching factor and emittances.

\section{References}

1. A. Hofmann and F. Pedersen, IEEE Trans. Nucl. Sci, NS-26, No. 3, 3526 (1979).

2. H. Schönauer, private communication.

3. U. Bigliani et al., IEEE Trans. Nucl. Sci. NS-18, No. 3, 233 (1971).

4. F. Pedersen, IEEE Trans. Nucl, Sci. NS-22, No. 3, 1906 (1975).

5. F.A. Ferger, W. Schne11, USSR 2nd Nat. Conf. on Particle Accelerators, Moscow, Sept, 1970.

6. K.W. Robinson, CEA, Report No. CEAL-1010 (Feb, 1964).

7. F. Pedersen and F. Sacherer, IEEE Trans. Nucl. Sci. NS-24, No. 3, 1396 (1977)

8. U. Bigliani, IEEE Trans. Nucl. Sci. NS-18, No. 3, 352 (1971) 